\title{
Low risk of adverse effects from epinephrine in hypertensive patients, but relevant high-quality literature is sparse
}

\author{
In people who have hypertension, does the use of epinephrine-containing \\ anaesthetic solution or epinephrine-impregnated retraction cord produce \\ adverse cardiovascular outcomes?
}

\begin{abstract}
Bader JD, Bonito AJ, Shugars DA. A systematic review of cardiovascular effects of epinephrine on hypertensive dental patients. Oral Surg Oral Med Oral Pathol 2002; 93:647-653
\end{abstract}

Objective To identify any additional risk of adverse cardiovascular outcomes in hypertensive patients from the use of epinephrinecontaining anaesthetic solution or epinephrine-impregnated retraction cord.

Data sources Medline, Embase and the Cochrane Controlled Trials Register were searched, along with reference lists from relevant studies. Study selection Only studies that had been conducted in a dental environment were selected. Those used included separate analysis of patients; administration of known concentrations of epinephrine, via block or infiltration local anaesthetic or impregnated retraction cord; and a wide range of haemodynamic and cardiovascular outcomes. Studies reporting on fewer than five hypertensive patients were excluded.

Data extraction and synthesis Data extraction was performed independently by two reviewers. The principle outcomes, ie, diastolic and systolic blood pressure and heart rate, were tabulated and the unweighted mean for three studies was calculated.

Results Six studies, representing 325 patients, met the inclusion criteria. No adverse outcomes were reported. Use of epinephrine in uncontrolled hypertensive patients was associated with small nonsignificant increases in blood pressure. Only one study examined controlled hypertensives. No studies addressed the effects of retraction cord.

Conclusions Although there is only a small increase in the risk of adverse effects in hypertensive patients when epinephrine is used, the quality and quantity of the relevant available literature is poor.
Address for correspondence: James D Bader, Sheps Centre, CB\#7590, School of Dentistry, University of North Carolina, Chapel Hill NC 27599, USA.

\section{Commentary}

Hypertension is a very common condition. In the US, it affects nearly a quarter of the adult population: in the United Kingdom around $10 \%$ of adult dental patients are hypertensive. In addition to problems created by the underlying disease, antihypertensive drug therapies can also impact on dental management. This review concentrated on the effects of epinephrine (adrenaline) administered in a dental setting, examining the cardiovascular responses of treated and untreated hypertensive patients.

Other than its use in the treatment of medical emergencies such as anaphylactic shock, epinephrine is administered in dentistry for two purposes. First, it is a constituent of dental local anaesthetic solutions. Secondly, it is used as a haemostatic agent in gingival retraction cord. It is possible, however, that epinephrine administered during dental treatment could increase an already high systolic blood pressure. This might lead to a hypertensive crisis causing a stroke or heart failure.

Six studies over a 42-year period satisfied the criteria for inclusion in this review. Only one of these investigated the effects of "dental" epinephrine in patients who were receiving treatment for hypertension. No study looking at the effects of epinephrine in gingival retraction cord was included. All patients were undergoing dental extractions or minor oral surgery.

The evidence suggested that diastolic blood pressure decreased and heart rate increased in both normotensive and hypertensive patients during dental treatment with epinephrinecontaining local anaesthetics. These changes were small and unlikely to be clinically significant. Systolic blood pressure rose slightly in hypertensive patients but not in normotensive individuals when epinephrine-containing local anaesthetics were injected.

One of the targets of the UK parliament's "The Health of the Nation" white paper of $1992^{1}$ was to reduce hypertension. Drug therapy for hypertension can impact on dental treatment including interactions with epinephrine. Non-potassium-sparing diuretics and calcium channel blockers can also exacerbate the reduction in plasma potassium produced by epinephrine. Beta-adrenergic blocking drugs can nullify the beta 2 vasodilatation produced by epinephrine leading to an uncompensated alpha-adrenergic increase in systolic blood pressure. It is therefore surprising and disappointing that only one study has investigated the effects of epinephrine in people who take antihypertensive therapy. Thus, there is little information concerning the safety of epinephrine at dental doses in these patients. 
The authors of this review are conscious of its limitations. A surprisingly small number of patients were included considering this was an investigation of an issue of concern to the dental profession for over 40 years. The dose of epinephrine in the local anaesthetics and the volume of anaesthetic delivered varied between investigations. The studies included used different recording devices. The timings of the recordings pre- and postinjection differed between trials and only one study used continuous recording of heart rate. Significant transient changes in cardiovascular responses may have been missed, therefore. Only heart rate and blood pressure were recorded in all of the studies and these are not the only indicators of cardiovascular risk. The increasing sophistication of recording devices should allow more complete monitoring of patients in future studies, however.

It is difficult to argue with the review's conclusion. The strength of the evidence about the effect of dental epinephrine on cardiovascular risk is poor because there has been no complete assessment of all the risk indicators.

\section{Practice Points}

- Epinephrine in dental local anaesthetics has little effect on blood pressure and heart rate in hypertensive patients at doses equivalent to one or two cartridges.

- There is little evidence concerning the safety of epinephrine in dental local anaesthetics for other cardiovascular risk indicators.

- There is no reliable evidence concerning the safety of epinephrine in gingival retraction cord in hypertensive patients.

\section{John G Meechan \\ School of Dental Sciences, University of Newcastle, Newcastle upon Tyne, UK}

1. Anon. The Health of the Nation: A Strategy for Health in England. Presented to Parliament by the Secretary of State for Health by Command of Her Majesty, July 1992. London: HMSO; 1992.

Evidence-Based Dentistry (2003) 4, 86-87.

doi:10.1038/sj.ebd.6400223 